\title{
Validation of fluorescent dust marking of Culicoides biting midges and the design of a self-marking technique
}

\author{
Georgette Kluiters $^{1 *}$, Kristina Hunter ${ }^{1}$ and Matthew Baylis ${ }^{1,2}$
}

\begin{abstract}
Background: Investigation of insect flight patterns frequently involves the use of dispersal studies. A common method for studying insect dispersal is mark-release-recapture (MRR) techniques using wild-caught insects in their natural environment; however, this requires a suitable marker. At present, no studies have been performed to identify markers that are suitable for use in midges within the Obsoletus Group, and visible by eye or down a light microscope.
\end{abstract}

Methods: A series of 11 experiments were undertaken to determine the effectiveness of three colours of Brilliant General Purpose (BGP) fluorescent dusts in marking Culicoides midges. Three areas were focused on: 1) dust properties, 2) the effect on Culicoides, and 3) dust application in the field.

Results: All three dusts were insoluble in water, 10\% washing-up liquid and 70\% ethanol. They were visible down a microscope, with and without the use of a black light, and two were highly visible without the need for a microscope. The dusts remained adherent to the marked Culicoides for the duration of the experiments, did not transfer between marked and unmarked individuals or the environment, and remained adherent when the Culicoides were stored in an ethanol or water-based solution. The dusts had no effect on the mortality rate of the insects over the $48 \mathrm{hrs}$ of the experiment. There were no significant differences between the recorded behaviours undertaken by undusted control Culicoides and the BGP fluorescent dusted Culicoides. Field-based marking of Culicoides can be achieved using a 'self-marking' technique, whereby the trapping vessel is pre-dusted with fluorescent dust prior to trapping the individuals to be marked.

Conclusions: This is the first study to identify BGP fluorescent dusts as markers for use with Obsoletus Group Culicoides. BGP fluorescent dusts provide a quick and effective method of marking and identifying Culicoides for both field and laboratory studies. The self-marking technique minimises the time needed to handle specimens prior to release.

Keywords: Culicoides, Mark-release-recapture, MRR, Dispersal, Fluorescent, Dust, Marking, Self-marking

\section{Background}

Culicoides flight behaviour is believed to drive the dispersal of midge-borne diseases, such as bluetongue (BT), from farm-to-farm; and modelling studies suggest that BT outbreaks cannot occur in the absence of local spread by midges [1]. Culicoides flight behaviour is

\footnotetext{
* Correspondence: g.kluiters@liverpool.ac.uk

${ }^{1}$ Liverpool University Climate and Infectious Diseases of Animals (LUCINDA) Group, Institute of Infection and Global Health, University of Liverpool, Leahurst Campus, Neston, Cheshire CH64 7TE, UK

Full list of author information is available at the end of the article
}

therefore a critical aspect of BT epidemiology, but it remains poorly understood [2].

Investigation of insect flight patterns frequently involves the use of dispersal studies [3-5]. Different approaches have been attempted to study the dispersal of Culicoides spp. midges: long-distance dispersal studies have utilised evidence from disease outbreaks [6-10], whereas shortdistance studies have involved direct capture of adult midges near breeding sites. An optimal approach would be a mark-release-recapture study (MRR) using wildcaught midges in their natural environment, however this 
is only possible with the availability of a suitable marking agent. Any selected marker must not have a detrimental effect on the survival or behaviour of marked specimens or the environment, but must also remain adherent under adverse conditions, be highly visible and allow differentiation between marked and unmarked individuals [11].

The Palaearctic BT vectors, members of the Obsoletus Group, are highly abundant throughout northern Europe [12], yet their dispersal ability has not been determined. Until now, dispersal studies have only been successfully undertaken on C. mississippiensis [4], C. mohave [13] and C. variipennis [14], as well as the Pulicaris Group in Denmark [15]. As such, investigation of marking agents has mainly been undertaken on these species. A recent study in Denmark investigated the use of fluorescein isothiocyanate (FITC) to mark Culicoides and successfully employed this marker in a MRR experiment [15]. The marker has a number of drawbacks, however, as it requires a plate scanner and associated software for the detection of FITC on individual specimens, is removed from Culicoides with the addition of ethanol, and is light-sensitive so could fade over time on Culicoides released in the field. The impact of FITC on the survival rate of Culicoides was also not tested, and spurious results may occur if Culicoides were to become contaminated with other autofluorescent matter, such as some types of pollen, in the field.

Although the use of micronized fluorescent dusts have produced good results in a laboratory setting [4], and also proved successful in field trials $[4,13,14]$, a number of problems still remain. Firstly, the use of easily identifiable fluorescent dusts that can be seen by eye have not been tested on Obsoletus Group members, now identified as major vectors of viruses in Europe. Secondly, the manufacturers of such dusts used in previous studies no longer exist.

The aim of this study was therefore to determine whether Obsoletus Group members could be marked effectively using micronized fluorescent dusts under both laboratory and field settings. Specific objectives included testing the hypothesis that the dusts have no adverse effects on Culicoides behaviour, life-span or flight compared to unmarked controls. The ability to detect marked individuals trapped in both water and ethanol was investigated, as well as whether any dust transfer occurs from marked individuals to other Culicoides or the environment. As no self-marking method currently exists for marking Culicoides in the field the final aim of this set of experiments was to explore the possibility of a self-marking technique. This paper is one of a pair of companion papers, the second of which uses the fluorescent dusts and the selfmarking method devised in this paper in a MRR study on Palaearctic Culicoides [16].

\section{Methods}

\section{Selection of fluorescent dusts}

Brilliant General Purpose (BGP) Fluorescent Pigments manufactured by Brilliant Group (San Francisco, USA) were selected as the fluorescent dusts to be trialled due to their small particle size (3-5 microns), wide range of colours which would be useful for repetitions of markrelease-recapture (MRR) experiments, non-toxic nature, and availability. The BGP series of fluorescent pigments are principally used in the coloration of paints, coatings, inks and plastics. Three colours were selected to be trialled as marking agents - pink (BGP-PK111), green (BGP-GR118) and yellow (BGP-YE117).

\section{Marking method}

In July 2010 the use of Brilliant General Pigment (BGP, Brilliant Group, Inc., San Francisco, USA) micronized fluorescent dusts in marking Culicoides for dispersal studies was investigated using a series of 11 laboratory studies falling under three areas of interest; 1) dust properties; 2) effect of dust on Culicoides; and 3) dust application. Culicoides were collected at the University of Liverpool's Leahurst Campus using an Onderstepoorttype down-draught black light trap placed at $2 \mathrm{~m}$ in height, and were stored in plastic trapping containers with a gauze bottom with a $10 \%$ sucrose solution embedded in cotton wool for sustenance (Figure 1). During the following experiments, midges were incapacitated on a cold plate set to $-15^{\circ} \mathrm{C}$ for 15 seconds to allow for the

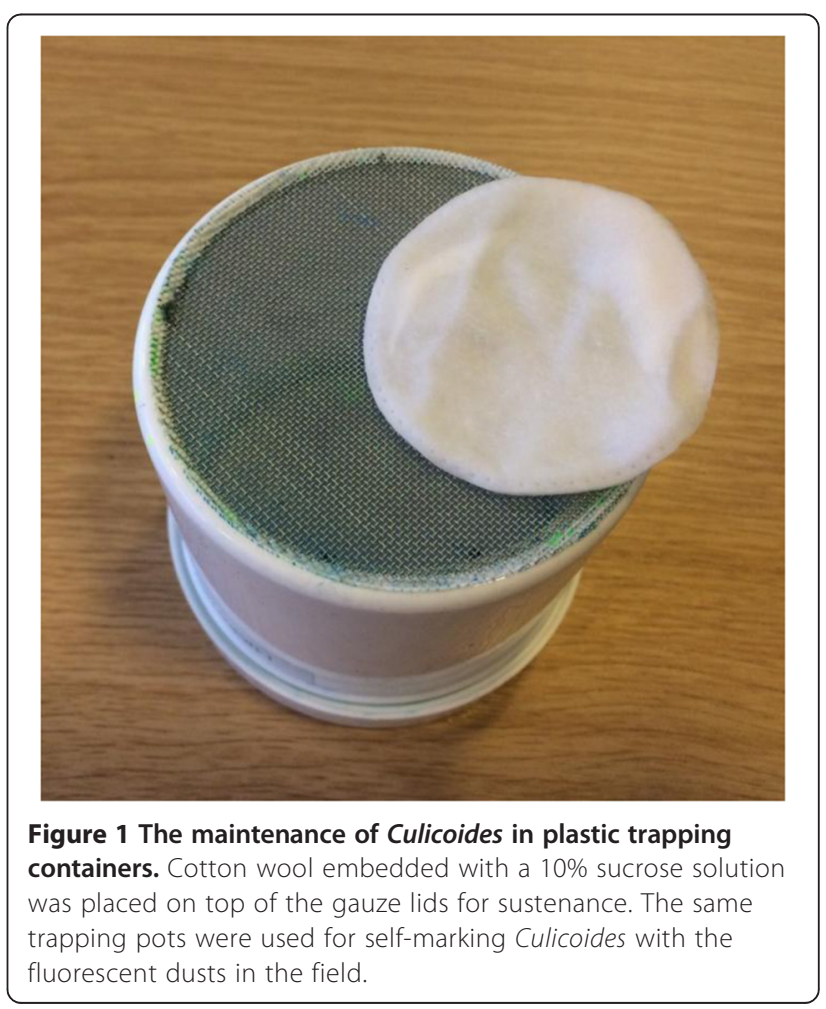


separation of members of the Obsoletus Group according to their wing patterns. Culicoides were killed following transfer to a $-80^{\circ} \mathrm{C}$ freezer for $20 \mathrm{~min}$.

\section{Investigation of dust properties}

The solubility of the dusts was determined in three test solutions of $100 \mathrm{ml}$ of water, a $10 \%$ detergent solution and $70 \%$ ethanol prepared in triplicate and held at $10^{\circ} \mathrm{C}$, $20^{\circ} \mathrm{C}$ and $30^{\circ} \mathrm{C}$. Exactly $1 \mathrm{~g}$ of each dust (green, pink and yellow) was added individually to the test solutions, and the solutions were agitated using a magnetic stirrer (Stuart Heat-stir SB162, Bibby Scientific Limited, UK) for 30 minutes. Dust solubility was assessed via filtration of beaker contents using $5 \mu \mathrm{m}$ filtration-paper. The tests were repeated using $0.01 \mathrm{~g}$ of each coloured dust.

Dust visibility was assessed on 30 killed Culicoides by dusting them with $0.01 \mathrm{~g}$ of each dust while stored in a $15 \mathrm{ml}$ tube, and then inspecting them using a stereomicroscope under both natural and black light, before live-dusted Culicoides were inspected from the experiments that followed.

Dust adherence was initially tested on 30 killed Culicoides which were added to $0.01 \mathrm{~g}$ of the marker in a $15 \mathrm{ml}$ tube, agitated with an electronic shaker and inspected for dust coverage, before being stored at $10^{\circ} \mathrm{C}$ and rechecked at 24 and $48 \mathrm{hrs}$. The experiment was repeated, with marked midges added to $10 \mathrm{ml}$ test solutions of water, $10 \%$ detergent solution or $70 \%$ ethanol, before being held at $10^{\circ} \mathrm{C}, 20^{\circ} \mathrm{C}$ or $30^{\circ} \mathrm{C}$ for $30 \mathrm{~min}$, after which time the Culicoides were then individually removed and examined for dust adherence. These specimens were further stored and rechecked at 24 and $48 \mathrm{hrs}$ and the solutions fluoresced under a blacklight to check for the present of fluorescent dust.

To determine dust transfer to the environment, 30 live Culicoides were transferred to a $15 \mathrm{ml}$ tube containing $0.01 \mathrm{~g}$ of fluorescent dust and the tube gently rolled to mark the insects. Marked Culicoides were transferred into gauze-ended trapping beakers with paper towellined bases for $48 \mathrm{hrs}$ before being killed and removed, and the paper towel and container surfaces inspected for dust transfer using a black light.

\section{Effects of dust on culicoides}

Dust toxicity was assessed by transferring 30 livemarked (using the tube rolling method above) and 30 unmarked Culicoides into gauze-ended trapping beakers and the mortality rate of both groups was recorded every $30 \mathrm{~min}$ for 4 hours, with Culicoides then rechecked at 24, 48 and $72 \mathrm{hrs}$. The effect of the dusts on Culicoides survival was determined by survival analysis, using the log-rank test, and presented as a Kaplan Meier graph.
Scan sampling was used to determine the impact of dusts on behaviour of 30 live-marked individuals against 30 unmarked control Culicoides held in separate containers. The proportion of midges exhibiting six listed behaviours (flying, climbing, walking, feeding, cleaning and resting) at $5 \mathrm{~min}$ intervals for the first hour, every $15 \mathrm{~min}$ for the second hour, and $30 \mathrm{~min}$ for the third hour was compared between the groups. The effect of the dusts on the behaviour Culicoides was assessed using chi-square analyses.

The transfer of fluorescent dust between Culicoides was assessed by placing 30 live-marked and 30 unmarked Culicoides into a gauze-ended trapping beaker for 24 hrs before being killed and inspected under a microscope.

\section{Dust application}

Three approaches to marking were compared to determine ease of application, evenness of coverage, mortality, injury and waste residue. The methods involved using a fine brush to sieve dust through the gauze surface of a trapping pot containing Culicoides, using a syringe to inject dust into a vacuum flask containing midges and, pre-dusting the inside surface of trapping pots [Figure 1 shows a trapping pot] to create a fine layer of dust before Culicoides were placed in the pots and allowed to walk independently over the surfaces.

Due to the success of the pre-dusted trapping pot method, pots were further pre-dusted with $0.1 \mathrm{~g}, 0.25 \mathrm{~g}$, $0.5 \mathrm{~g}, 0.75 \mathrm{~g}$ or $1 \mathrm{~g}$ of dust and attached to an OVI trap at the University of Liverpool's Leahurst Campus where the trap was run overnight and the proportion of marked midges determined the following day. The mortality rate of the Culicoides collected overnight was determined using chi-square analysis.

\section{Results}

Investigation of dust properties

Both $0.01 \mathrm{~g}$ and $1 \mathrm{~g}$ of the three fluorescent dusts were insoluble in water, $10 \%$ washing-up liquid solution and ethanol at 10,20 and $30^{\circ} \mathrm{C}$. The dusts were visible, both directly and under a stereomicroscope in natural or black-light conditions, with no variation in visibility detected. They were visible on all surfaces dusted, with no difference in visibility identified.

The three dust markers adhered to all midges for the duration of the experiment ( 48 hours). BGP yellow and green dusts demonstrated greatest attachment to the wings, wing base and legs, while BGP Brilliant Pink adhered to the thorax. Five of the killed Culicoides marked by agitating the pre-dusted tubes on an electronic shaker showed damage to their wings and antennae, while Culicoides marked via rotation of the tube exhibited no such damage. 
Following storage of the killed and marked Culicoides in water, $10 \%$ detergent solution, and $70 \%$ ethanol for $48 \mathrm{hrs}$, the dusts remained adherent on the individuals and no extraneous particles were found either on the container or liquid they were stored in. The results did not vary with temperature. No transfer occurred with any dust from marked midges to any of the test surfaces over the 48 hours of the experiment.

\section{Effect of dust on Culicoides}

There were no differences in the survival between the un-dusted control Culicoides and those dusted with pink, yellow or green fluorescent dust (log-rank test, $P=0.914$ ). Kaplan Meier survival curves for the dusts can be seen in Figure 2.

Overall there were no significant differences between the behaviours undertaken between the undusted control Culicoides and the BGP fluorescent dusted Culicoides (chi-square, $P=0.922$ ). Differences were observed between the three control populations of unmarked Culicoides (chi-square, $P \leq 0.001$ ). The percentage of Culicoides exhibiting the listed behaviours during the experiment can be seen in Figure 3.

No dust transfer occurred between dust-marked Culicoides and unmarked controls, with 30 marked Culicoides and 30 unmarked control Culicoides identified using a stereomicroscope at the end of the experiment.

\section{Dust application}

Of the three dust application methods trialled in the laboratory (fine brush technique, syringe injection, predusted containers), the syringe-based method proved the most difficult in terms of accurately measuring the required amount of dust for application, also leading to wastage with dust remaining in the syringe. This method also led to a limited number of individuals being marked within the time limit of the experiment (14 individuals out of 30). Four individuals also exhibited injury to their wings when this method was employed.

Although the required amount of dust was easy to measure when using the fine brush technique, it was difficult to obtain an even coverage of individuals through the mesh gauze of the trapping pot, with some individuals receiving a higher coverage of dust than others (although all 30 midges were marked). The marking method worked best when the pot itself was already pre-dusted with fluorescent dust prior to the Culicoides being added (all 30 Culicoides marked). There was no mortality using any method.

The number of Culicoides trapped during each replicate of the field-marking experiment can be seen in Table 1. The total Culicoides trapped during the 5 consecutive trapping nights was 880 . Of these, $100 \%$ of individuals were marked each night, irrespective of the weight of dust used to pre-dust the container. The mortality rate of Culicoides did not vary significantly between different dust weights (chi-square, $P=0.1$ ).

\section{Discussion}

This study successfully identifies a fluorescent dust marker and self-marking technique for members of the Obsoletus Group of Culicoides, the vectors of bluetongue virus in northern Europe. To our knowledge, there have been no studies published on the use of

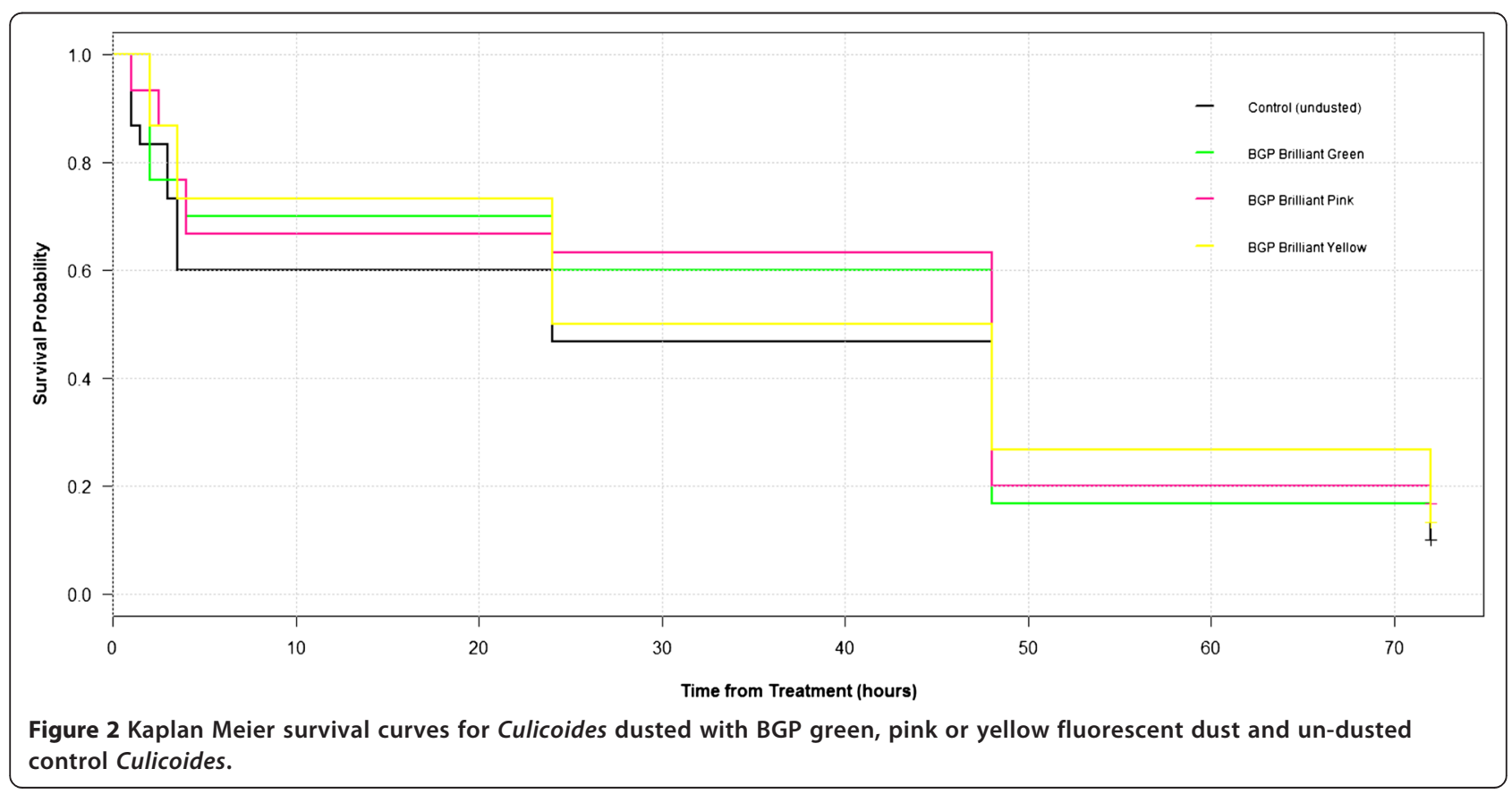



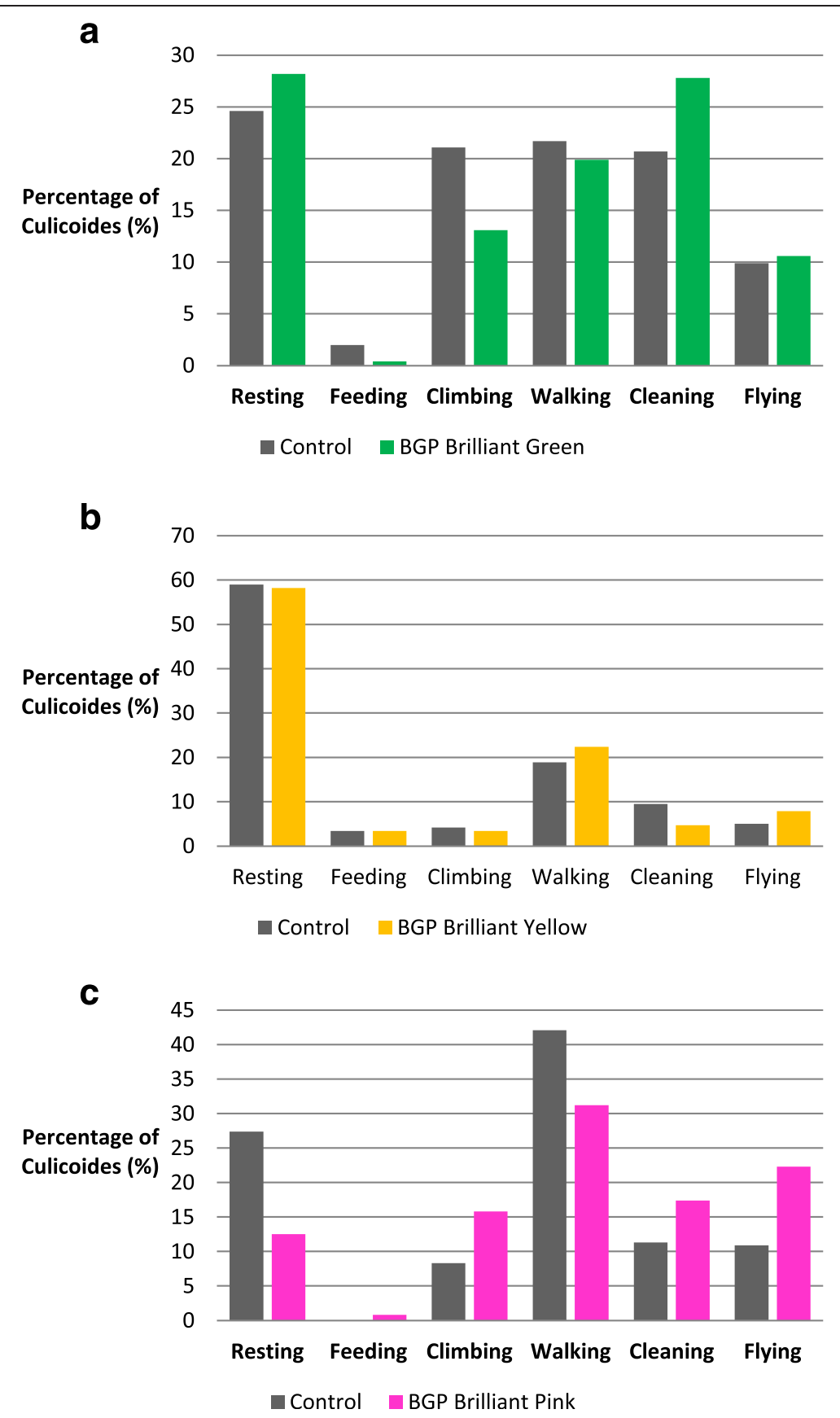

Figure 3 Percentage of Culicoides exhibiting six observed behaviours when dusted with fluorescent dusts in comparison to un-dusted control Culicoides; where a) uses Brilliant General Purpose (BGP) Green fluorescent dust; b) BGP Yellow fluorescent dust; and c) BGP Pink fluorescent dust.

fluorescent dusts for marking Culicoides since the 1980s $[4,13,14]$, and the fluorescent dusts employed in these early studies are no longer available. This technique should be useful in studying the dispersal behaviour of members of the Obsoletus Group under field conditions.

The dust properties tested indicate that the fluorescent dusts could be successfully used in mark-releaserecapture (MRR) experiments. As all dusts remained insoluble in water, $10 \%$ detergent solution, and $70 \%$ ethanol this would allow trapping during field investigations to be undertaken using standard trapping solutions of water with a drop of washing-up liquid in order to break the surface tension. It would also allow storage of insects in $70 \%$ ethanol solutions following collection. Similarly, the adherence experiments suggested that not only are the dusts insoluble in these test solutions, but they also remain adhered to the Culicoides themselves while in solution. As adherence to Culicoides, when 


$\begin{aligned} & \text { Table } \mathbf{1} \text { The number of Culicoides trapped, marked and } \\
& \text { dead when caught overnight in trapping containers } \\
& \text { pre-dusted with differing quantities of fluorescent dusts }\end{aligned}$
\begin{tabular}{llll}
\hline $\begin{array}{l}\text { Weight of } \\
\text { dust (g) }\end{array}$ & $\begin{array}{l}\text { Number of } \\
\text { culicoides } \\
\text { trapped }\end{array}$ & $\begin{array}{l}\text { Number of } \\
\text { culicoides marked }\end{array}$ & $\begin{array}{l}\text { Number of dead } \\
\text { culicoides }\end{array}$ \\
\hline $\mathbf{0 . 1}$ & 134 & 134 & 0 \\
$\mathbf{0 . 2 5}$ & 217 & 217 & 2 \\
$\mathbf{0 . 5}$ & 203 & 203 & 0 \\
$\mathbf{0 . 7 5}$ & 167 & 167 & 2 \\
$\mathbf{1 . 0}$ & 159 & 159 & 4 \\
\hline
\end{tabular}

stored in ethanol, was only evaluated for $48 \mathrm{hrs}$ in this experiment, more work would need to be undertaken to determine if long-term storage in ethanol would affect the adherence of the dusts.

Dust visibility was not reduced after the marked Culicoides were stored in solution. The use of a fluorescent light was not always required to detect marked individuals, as the pink and green fluorescent dusts were easily identifiable on the Culicoides without being fluoresced. The use of these dusts therefore provides a quickly identifiable marker, eliminating the need for more complex and time-consuming detection methods.

Transfer of fluorescent dust to the environment in which the marked Culicoides were held was not observed over the 24 hour period they were examined for. As it is likely that any superfluous dust would have been removed by the Culicoides rapidly, it is unlikely that dust transfer would occur at any time after this 24 hour period. Other researchers have incorporated dusts with gum arabic, so that particles could not easily be removed with preening and wing movements $[17,18]$, but that was not necessary here.

While visibility and adherence of the marker is of utmost importance, any selected marker must also not have a detrimental effect on the survival or behaviour of marked specimens [11]. Although an increase in mortality of marked Culicoides was observed over time, a similar increase was also observed in the unmarked controls.

Of the markers tested, there were no significant differences between the behaviours of the un-marked controls and the marked Culicoides, highlighting that application of the dusts as a marking agent in the field would not affect the results obtained by MRR experiments. Differences were observed, however, between the populations of control Culicoides used for each pair of experiments and this is likely to be due to the pairs of experiments being undertaken on different days. Although environmental conditions, such as temperature, were kept stable within the laboratory, the differences between days may be linked to different trapping conditions on each night (e.g. temperature or rainfall), the length of time
Culicoides were held in the live-trapping containers prior to the start of the behaviour experiment, or the number of Culicoides trapped in the live-trapping pot over night.

Although many important aspects of a marking agent were tested in this study, the laboratory environment within which the experiments were undertaken is unlikely to be identical to the conditions Culicoides would encounter in the field. We did not assess the impact of rain or wind on the adherence of the dusts to Culicoides, or their subsequent transfer to the environment. Similarly, the duration of these experiments was limited due to the difficulties encountered with keeping wild-caught Obsoletus Group members alive in a laboratory environment. Although laboratory colonies of other Culicoides species are available, the behaviours of these individuals may be markedly different from those of their wild counterparts and as a colony of Obsoletus Group members has not been established, wild-caught individuals were employed here.

Following the series of laboratory experiments on the dusts a small field-trial of a self-marking method was successfully employed. The field-study highlighted the ease of use of the pre-dusted trapping pot method in self-marking Culicoides for MRR studies, eliminating the need to manually apply dust to the insects the following morning. As no difference in mortality rates or dust coverage of Culicoides occurred when different quantities of dusts were applied to the pots, we recommend that a sufficient quantity of dust is applied so as to fully cover the sides and gauze bottom of the trapping pot, with this quantity varying depending on the size of trapping vessel used.

Relatively small numbers of Culicoides were trapped during the field-trial so it would be useful to replicate the trial in an area where larger numbers of insects are likely to be trapped to determine whether dust coverage remains at $100 \%$ with large numbers of insects. A MRR study was undertaken in a companion paper using the BGP fluorescent dusts and the self-marking method described here [16].

\section{Conclusions}

This is the first study to identify BGP fluorescent dusts as markers for use with Obsoletus Group Culicoides. BGP fluorescent dusts are a suitable marking agent for Culicoides midges as they do not influence either survival or flight behavior of Obsoletus Group members in the laboratory. Marked midges remained distinguishable for their entire lifetime during the experiment; dusts did not transfer from marked to unmarked individuals or the environment; the mortality rate of marked midges did not differ from controls under laboratory conditions; and, importantly for trapping and storing Culicoides, the 
dust did not dissolve or wash off in either ethanol or water. Pre-dusting trapping pots with BGP fluorescent dusts prior to trapping provides a fast and reliable method for self-marking Culicoides in the field and should prove useful for MRR studies.

\section{Abbreviations}

BGP: Brilliant General Purpose; MRR: Mark-release-recapture.

\section{Competing interests}

The authors declare that they have no competing interests.

\section{Authors' contributions}

GK conceived and designed the study. GK and MB were awarded a BBSRCfunded Summer Studentship which enabled KH to support the laboratory studies. GK \& KH undertook the laboratory investigations and performed the analyses. GK wrote the first draft of the manuscript. All authors contributed to the final version of the manuscript. All authors read and approved the final manuscript.

\section{Acknowledgements}

This study is part of a BBSRC-funded PHD carried out by GK under the supervision of MB. A BBSRC Vacation Bursary provided funding for $\mathrm{KH}$.

\section{Author details}

${ }^{1}$ Liverpool University Climate and Infectious Diseases of Animals (LUCINDA) Group, Institute of Infection and Global Health, University of Liverpool, Leahurst Campus, Neston, Cheshire CH64 7TE, UK. ${ }^{2}$ National Institute for Health Research, Health Protection Research Unit in Emerging and Zoonotic Infections, University of Liverpool, Leahurst Campus, Neston, Cheshire CH64 7TE, UK.

Received: 4 December 2014 Accepted: 12 January 2015

Published online: 27 January 2015

\section{References}

1. Turner J, Bowers RG, Baylis M. Modelling bluetongue virus transmission between farms using animal and vector movements. Sci Rep. 2012;2:319. doi: 10.1038/Srep00319.

2. Carpenter S, Wilson A, Mellor PS. Culicoides and the emergence of bluetongue virus in northern Europe. Trends Microbiol. 2009;17(4):172-8.

3. Danthanarayana W. Insect flight. Dispersal and migration. Entomol Exp Appl. 1986;48:301-2

4. Lillie T, Kline D, Hall D. The dispersal of Culicoides mississippiensis (Diptera: (eratopogonidae) in a salt marsh near Yankeetown, Florida. J Am Mosq Control Assoc. 1985;1:463-7.

5. Valerio L, Facchinelli L, Ramsey JM, Bond JG, Scott TW. Dispersal of male Aedes aegypti in a coastal village in southern Mexico. Am J Trop Med Hyg. 2012;86(4):665-76.

6. Burgin LE, Gloster J, Sanders C, Mellor PS, Gubbins S, Carpenter S. Investigating incursions of bluetongue virus using a model of long-distance Culicoides biting midge dispersal. Transbound Emerg Dis. 2013;60:263-72.

7. Ducheyne E, De Decken R, Becu S, Codina B, Nomikou K, Mangana-Vougiaki $\mathrm{O}$, et al. Quantifying the wind dispersal of Culicoides species in Greece and Bulgaria. Geospat Health. 2007;2:177-89.

8. Eagles D, Deveson T, Walker PJ, Zalucki MP, Durr P. Evaluation of long-distance dispersal of Culicoides midges into northern Australia using a migration model. Med Vet Ent. 2012;26:334-40.

9. Hendrickx G, Gilbert M, Staubach C, Elbers A, Mintiens K, Gerbier G, et al. A wind density model to quantify the airborne spread of Culicoides species during north-western Europe bluetongue epidemic. Prev Vet Med. 2008:87:162-81.

10. Sedda L, Brown HE, Purse BV, Burgin L, Gloster J, Rogers DJ. A new algorithm quantifies the roles of wind and midge flight activity in the bluetongue epizootic in northwest Europe. Proc R Soc B, Biological Sciences. 2012;279(1737):2354-62.

11. Hagler JR, Jackson CG. Methods for marking insects: current techniques and future prospects. Annu RevEntomol. 2001;46:511-43.
12. Kluiters $G$, Sugden $D$, Guis $H$, McIntyre KM, Labuschagne $K$, Vilar MJ, et al Modelling the spatial distribution of Culicoides biting midges at the local scale. J App Ecol. 2013;50:232-42.

13. Brenner R, Wargo M, Stains G, Mulla M. The dispersal of Culicoides mohave (Diptera: Ceratopogonidae) in the desert of southern California. Mosq News. 1984:44:343-50.

14. Lillie T, Marquardt W, Jones $R$. The flight range of Culicoides variipennis (Diptera: Ceratopogonidae). Can Entomol. 1981;113:419-26.

15. Kirkeby C, Bødker R, Stockmarr A, Lind P, Heegaard PMH. Quantifying dispersal of European Culicoides (Diptera: Ceratopogonidae) vectors between farms using a novel mark-release-recapture technique. PLoS One. 2013;8:e61269.

16. Kluiters G, Swales H, Baylis M. Local dispersal of Palaearctic Culicoides biting midges estimated by mark-release-recapture. Parasit Vectors. 2015;8:54

17. Sinsko MJ, Craig GB. Dynamics of an isolated population of Aedes triseriatus (Diptera: Culicidae). I. Population size. J Med Entomol. 1979;15:89-98.

18. Brust RA. Dispersal behaviour of adult Aedes sticticus and Aedes vexans (Diptera: Culicidae) in Manitoba. Can Entomol. 1980;112:31-42.

\section{Submit your next manuscript to BioMed Central and take full advantage of:}

- Convenient online submission

- Thorough peer review

- No space constraints or color figure charges

- Immediate publication on acceptance

- Inclusion in PubMed, CAS, Scopus and Google Scholar

- Research which is freely available for redistribution

Submit your manuscript at www.biomedcentral.com/submit 